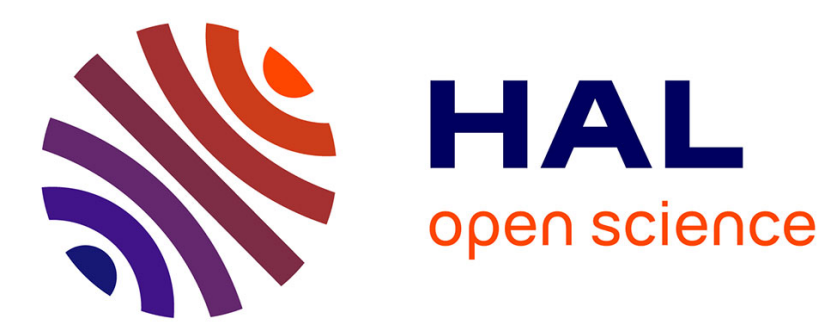

\title{
Critical properties of cluster size distribution in an asymmetric diffusion-aggregation model
}

\author{
Jean-Yves Fortin
}

\section{To cite this version:}

Jean-Yves Fortin. Critical properties of cluster size distribution in an asymmetric diffusion-aggregation model. Physical Review E , 2019, 100, pp.052108. 10.1103/PhysRevE.100.052108 . hal-02325245v2

\section{HAL Id: hal-02325245 \\ https://hal.science/hal-02325245v2}

Submitted on 6 Nov 2019

HAL is a multi-disciplinary open access archive for the deposit and dissemination of scientific research documents, whether they are published or not. The documents may come from teaching and research institutions in France or abroad, or from public or private research centers.
L'archive ouverte pluridisciplinaire HAL, est destinée au dépôt et à la diffusion de documents scientifiques de niveau recherche, publiés ou non, émanant des établissements d'enseignement et de recherche français ou étrangers, des laboratoires publics ou privés. 


\title{
Critical properties of cluster size distribution in an asymmetric diffusion-aggregation model
}

\author{
Jean-Yves Fortin * \\ Department of Physics and Astronomy \\ Seoul National University, Seoul 08826, South Korea ${ }^{\dagger}$
}

\begin{abstract}
We consider a stochastic dynamics for a system of diffusing hard-core particles on a periodic chain with asymmetric diffusion rules. A given cluster of particles can diffuse to the right as a whole but the particle located on the left boundary of the cluster is allowed to break-off from it and diffuse to the left. Clusters of particles can eventually merge with other clusters. These rules allow for the creation of clusters of different sizes. We discuss the size distribution of the clusters in the long time or steady state limit, as a function of the particle concentration $c$. We consider the general time dependent master equation based on Smoluchowski's theory for local cluster merging or fragmentation and diffusion processes, and study the solutions using the generating function in the large size limit. We found that there exists a critical density $c^{*}=\sqrt{2}-1$ for which the cluster distribution decays like a power law with exponent $5 / 2$.

PACS numbers: 05.10.Gg,05.20.Dd,05.40.-a,05.50.+q
\end{abstract}

\section{INTRODUCTION}

Statistical properties of coalescence and fragmentation processes have been studied in various fields of physics and chemistry [1-7] where for example chemical constituents react to form new molecules, or fragment into elementary units. Both aggregation and fragmentation

\footnotetext{
* Permanent address: Laboratoire de Physique et Chimie Théoriques, CNRS UMR7019, Université de Lorraine, 1 Boulevard des Aiguillettes, BP 7023954506 Vandoeuvre-lès-Nancy Cedex

${ }^{\dagger}$ Electronic address: jean-yves.fortin@univ-lorraine.fr
} 
reactions are governed by stochastic processes, influenced eventually by two-body collision properties [8, 9]. The kinetic of such processes is described by a Smoluchowski's master equation [10], which is a balance equation for determining the particle distribution in size or mass in the steady or equilibrium state $[11,12]$, and can be used for example to determine the size of interstellar dust grains [13] or even particle size in Saturn rings [14].

The dynamics is usually controlled by reaction rates depending on the size or mass of the particles, and it is assumed in most of Smoluchowski's models that both aggregation and fragmentation rates follow a scaling law: they depend intrinsically on the sum or product of the individual mass or size of the merging components with some power exponent. Therefore a general description of the physical properties such as mass or size distribution depends indeed on the microscopic details of those kernels $[5,11,15,16]$. For example, in the case of gravitational systems, the aggregation rate is proportional to the sum of the constituent masses, with eventually some power law, whereas in the case of branched polymerization they are proportional to the product of the molecule weights. In particular, the size distribution when the rate is constant and when a monodisperse source is introduced exhibits, in addition to an exponential damping factor, a power law with a decay exponent equal to $3 / 2+\beta$ [11], $\beta$ being the weight exponent in the kernel.

In this paper we present a dynamical model of hard-core particles on a periodic chain of size $N$ and which can diffuse or fragment from or merge with other clusters. We can view the clusters of particles as polymers confined on a one-dimensional (1D) chain and which can react with the other neighboring clusters by chemical reaction. The dynamics we consider is defined more precisely by the following asymmetric and nonlocal rules: We assume that, at every time step, a particle located at the left boundary of a cluster can break-off from it at rate $1 / \tau$, and/or eventually aggregate with another cluster. A whole cluster can move to the right by one elementary step with the same rate, and eventually fuses with a neighboring cluster if present. The schematic process is displayed in Fig. 1. From these two processes emerge clusters of small and large sizes. Diffusion of large clusters in one direction allows them to grow in size by merging with other clusters, whereas their partial fragmentation on the opposite direction increases the number of small clusters. Overall, we expect that the size distribution will display a tail that decays like a power law. Also, the structure of the distribution will depend crucially on the particle concentration. The periodic boundary conditions are important for the asymmetric dynamics we consider, as they allow for the 
current of particles, due to the asymmetry of the dynamics, to flow along the chain and particles to be transferred to the other side once they reach a boundary.

The dynamics is microscopically irreversible and does not reach an equilibrium state [17], but there is a steady state as we will see below from which a well defined size distribution exists. This is due in particular to the fact that one-particle clusters are formed constantly by fragmentation of larger clusters, in absence of any source, and therefore there is no absorbing state. A total aggregation process would otherwise lead to a unique cluster, a phenomena known as gelation [18-20] where the largest cluster is the final state.

We can relate this dynamics to a traffic flow problem [21], where for example groups (clusters) of vehicles coherently move to the right, the last vehicle of the groups being allowed to slow down and detach from its cluster. A particle current is present and oriented to the right direction as only one-particle clusters can move to the left. This kind of dynamics can also be seen as a variation of the TASEP (Totally Asymmetric Exclusion Process) model [22], with non-local or coherent motion involved as clusters of particles can move collectively. The exclusion process comes from the fact that there is at most one particle per site (hard core interaction), and therefore individual particles cannot move if the neighboring sites are already occupied, like the ASEP model or Asymmetric Simple Exclusion Process.

The aim of this study is to show that this particular asymmetric fragmentation or merging process induces criticality in the cluster size distribution for a special concentration value. The master equation that we define in the next section is closely related to a non-linear differential-integral Smoluchowski's equation but with the constraint of dimensionality and space: Distances and therefore concentration between clusters influence the rate of merging.

From a technical point of view, many models based on Smoluchowski's equation can be solved exactly using generating functions [11, 23, 24] which are an adequate tool to obtain distribution moments. However the confinement effect in the present model renders the analysis more complicated as momentum interaction occurs in the Fourier space.

\section{MASTER EQUATION FOR CLUSTER DISTRIBUTION}

The stochastic process presented in the Introduction is characterized by a master equation. We first need to define the local probability for each $n$-particle cluster at a given time $t$ and location on the lattice, which is a succession of particles surrounded by two empty 

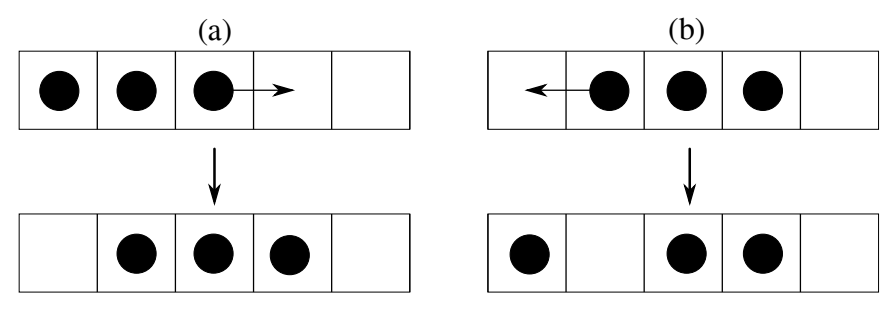

FIG. 1: Elementary processes with three particles. (a) Clusters can move to the right only. (b) The particle located on the left boundary can detach from the cluster and diffuse on the left.

sites:

$$
P_{n}(r)=\operatorname{Prob}\left(\circ \bullet^{r} \cdot{ }^{r+n-1} \bullet\right)
$$

where the first particle of the cluster is located at site $r \in\{1, \cdots, N\}$. The system is periodic with $P_{n}(r+N)=P_{n}(r)$, the number of clusters of size $n$ is $N_{n}=\sum_{r} P_{n}(r)$, and the total number of clusters $N_{c}=\sum_{n} N_{n}$. The number of particles is conserved, and equal to

$$
N_{p}=\sum_{r} \sum_{n} n P_{n}(r)
$$

and their concentration is denoted by $c=N_{p} / N$, with maximum cluster or cutoff size $N_{p}$. There are different processes which contribute to the change of probability $P_{n}(r)$ after a small time interval $\Delta t$. In the following, we note $\partial P_{n} / \partial t$ the rate of change of the probability when $\Delta t \rightarrow 0$. The first process $(a)$ is the decay or destruction of the cluster configuration by breaking-off and diffusion of the leftmost particle or diffusion of the whole cluster to the right

$$
\left(\frac{\partial P_{n}}{\partial t}\right)_{a}=-\operatorname{Prob}\left(\stackrel{\curvearrowleft}{\circ} \cdots^{r+n-1} \bullet\right)-\operatorname{Prob}(\circ \stackrel{r}{\bullet} \ldots \stackrel{r+n-1}{\bullet} \circ)
$$

Another decay is provided by the aggregation of neighboring clusters on the left, and particles on the right, processes $(b)$ and $(c)$

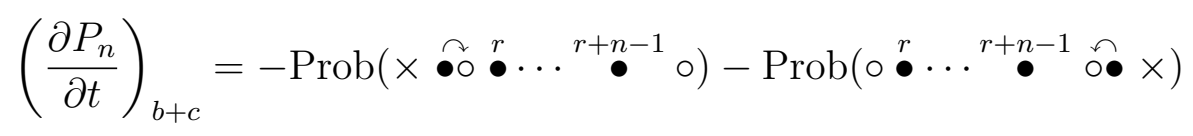

where the symbol $(\times)$ represents a site occupied or not by a particle. The positive contributions to $P_{n}$ come from a series of two-clusters aggregations by avalanches, process $(d)$

$$
\begin{aligned}
& \left(\frac{\partial P_{n}}{\partial t}\right)_{d}=\operatorname{Prob}\left(\circ^{r-1} \bullet \bullet .^{r+n-1} \bullet\right)+\operatorname{Prob}\left(\circ^{r-1} \bullet \bullet \bullet \ldots{ }^{r+n-1} \bullet\right)+\cdots \\
& +\operatorname{Prob}\left(\circ^{r-1} \bullet \cdots \odot^{r+n-1} \circ\right)
\end{aligned}
$$


The cluster can also be generated by one-particle diffusion from the right, process $(e)$

$$
\left(\frac{\partial P_{n}}{\partial t}\right)_{e}=\operatorname{Prob}\left(\circ \bullet \cdots^{r+n-2} \bullet \curvearrowleft \bullet \times\right)
$$

Process $(f)$ is the avalanche contribution of the same cluster originally located at $r-1$ and moving to the right

$$
\left(\frac{\partial P_{n}}{\partial t}\right)_{f}=\operatorname{Prob}\left(\circ^{r-1} \bullet \stackrel{r+n-2}{\bullet} \circ \circ\right)
$$

The last process $(g)$ consists in creating the cluster from a cluster of size $n+1$ from which the first particle separates by diffusing to the left hand side

$$
\left(\frac{\partial P_{n}}{\partial t}\right)_{g}=\operatorname{Prob}\left(\circ \stackrel{\curvearrowleft}{r^{-1}} \ldots{ }^{r+n-1} \bullet\right)
$$

The analytical expressions of the previous processes are given by products of cluster size distributions and their convolution, in the case where several clusters are contributing to the dynamics, and which are detailed in Appendix A. Overall, all the changes in $P_{n}(r)$ can be summed up and we obtain for $n \geq 2$ the time evolution:

$$
\begin{aligned}
\tau \frac{\partial P_{n}}{\partial t} & =-2 P_{n}(r)-P_{n}(r) \sum_{m=1}^{N_{p}-n} P_{m}(r-m-1)-P_{n}(r) \sum_{m=1}^{N_{p}-n} P_{m}(r+n+1) \\
& +\sum_{m=1}^{n-1} P_{m}(r-1) P_{n-m}(r+m)+P_{n-1}(r) \sum_{m=1}^{N_{p}-n+1} P_{m}(r+n) \\
& +P_{n}(r-1)\left[1-\sum_{m=1}^{N_{p}-n} P_{m}(r+n)\right]+P_{n+1}(r-1)
\end{aligned}
$$

For the particular case $n=1$, there is no $(d)$ process, and the $(e)$ process is replaced by a new process $\left(e^{\prime}\right)$ which can be written as

$$
\begin{aligned}
\left(\frac{\partial P_{1}}{\partial t}\right)_{e^{\prime}} & =\operatorname{Prob}(\stackrel{\substack{r \\
\bullet}}{\bullet} \times)=\frac{1}{\tau} \operatorname{Pr}(\circ \stackrel{r}{\circ} \bullet \times) \\
& =\frac{1}{\tau}\left[\sum_{m=1}^{N_{p}} P_{m}(r+1)-\sum_{m=1}^{N_{p}-1} P_{m}(r+1) \sum_{m^{\prime}=1}^{N_{p}-m} P_{m^{\prime}}\left(r-m^{\prime}\right)\right]
\end{aligned}
$$

It is convenient to introduce the generating function in the Fourier space for Eq.(8) and space average the probability distribution $\bar{P}_{n}$,

$$
\begin{aligned}
\hat{G}(x, k) & =\sum_{r} e^{-i k r} \sum_{n \geq 1} x^{n} P_{n}(r)=\sum_{n \geq 1} x^{n} \hat{P}_{n}(k), \\
\bar{P}_{n} & =\frac{N_{n}}{N}=N^{-1} \hat{P}_{n}(0)
\end{aligned}
$$


with momenta $k=2 \pi m / N, m=0, \cdots, N-1$. From this definition, we have the relations

$$
N_{c}=\hat{G}(1,0), \quad N_{p}=\partial_{x} \hat{G}(1,0)
$$

The generating function satisfies a master equation which incorporates all the previous processes, as well as the one-particle dynamics. Applying the transformation Eq.(10) to Eq.(8), we obtain

$$
\begin{aligned}
\tau \frac{\partial}{\partial t} \hat{G}(x, k) & =\left[-2+\left(1+x^{-1}\right) e^{-i k}\right] \hat{G}(x, k)-\frac{1}{N} \sum_{k^{\prime}} e^{-i\left(k-k^{\prime}\right)} \hat{G}\left(x, k^{\prime}\right) \hat{G}\left(e^{-i\left(k-k^{\prime}\right)}, k-k^{\prime}\right) \\
& +(x-1) \frac{1}{N} \sum_{k^{\prime}} e^{i\left(k-k^{\prime}\right)} \hat{G}\left(x e^{i\left(k-k^{\prime}\right)}, k^{\prime}\right) \hat{G}\left(1, k-k^{\prime}\right) \\
& +\frac{1}{N} \sum_{k^{\prime}} e^{-i k^{\prime}} \hat{G}\left(x e^{i\left(k-k^{\prime}\right)}, k^{\prime}\right)\left[\hat{G}\left(x, k-k^{\prime}\right)-\hat{G}\left(1, k-k^{\prime}\right)\right] \\
& +x e^{i k} \hat{G}(1, k)-x \frac{1}{N} \sum_{k^{\prime}} e^{i k^{\prime}} \hat{G}\left(1, k^{\prime}\right) \hat{G}\left(e^{-i\left(k-k^{\prime}\right)}, k-k^{\prime}\right)-e^{-i k} \hat{P}_{1}(k)
\end{aligned}
$$

with $\hat{P}_{1}(k)=\left.\partial_{x} \hat{G}(x, k)\right|_{x=0}$. We should notice that we assume $N \gg 1$ and that the finite size cutoff at $n=N_{p}$ in Eq.(8) is not taken into account in the expression for $\hat{G}(x, k)$. It can be checked from the previous equation that the number of particles is conserved, by computing directly $\partial_{t} N_{p}=\partial_{t} \partial_{x} \hat{G}(1,0)=0$. The last term in Eq.(12) corresponds to the contribution of one-particle clusters by fragmentation of bigger clusters. Since there is a continuous production of one-particle clusters, we expect the dynamics to reach a steady state. In particular, the time evolution of the cluster number $N_{c}=\hat{G}(1,0)$ is equal to

$$
\tau \frac{\partial}{\partial t} \hat{G}(1,0)=-2 \frac{1}{N} \sum_{k^{\prime}} e^{i k^{\prime}} \hat{G}\left(1, k^{\prime}\right) \hat{G}\left(e^{i k^{\prime}},-k^{\prime}\right)+\hat{G}(1,0)-\hat{P}_{1}(0)
$$

The dynamics is non-local as $\hat{G}(1,0)$ depends on non-zero momentum contributions. Let us consider the steady state of Eq.(12). If we assume the dynamics is governed by low momenta close to zero, we may try to study more precisely solutions given by $\hat{G}(x, k)=N \delta_{k, 0} \hat{G}_{0}(x)$ which is a mean-field approximation. This approximation will be compared later with the numerical results. The functional equation Eq.(12) can be solved for this solution, which yields

$$
\begin{aligned}
\hat{G}_{0}(x) & =\frac{1}{8 x}\left[4(1-x) \sqrt{1-\alpha x+\beta x^{2}}-4+\left(7-3 \sqrt{1-8 N^{-1} \hat{P}_{1}(0)}\right) x\right. \\
& \left.+\left(\sqrt{1-8 N^{-1} \hat{P}_{1}(0)}-1\right) x^{2}\right], \\
\alpha & =\frac{3}{2}\left(1-\sqrt{1-8 N^{-1} \hat{P}_{1}(0)}\right), \beta=\frac{1}{8}\left(1-8 N^{-1} \hat{P}_{1}(0)-\sqrt{1-8 N^{-1} \hat{P}_{1}(0)}\right)
\end{aligned}
$$


The value of $\hat{P}_{1}(0)$ is determined by the condition of particle conservation $\hat{G}_{0}^{\prime}(1)=c$. Solving this condition leads to the following expressions for $\hat{P}_{1}(0)$ and coefficients $\alpha$ and $\beta$ as function of $c$

$$
\begin{aligned}
\hat{P}_{1}(0) & =\frac{c(1-c)\left(1-c+2 c^{2}\right)}{(1+c)^{2}} N, \\
\alpha & =\frac{6 c(1-c)}{1+c}, \beta=\frac{c^{2}(1-c)^{2}}{(1+c)^{2}}, \frac{\alpha^{2}}{\beta}=36
\end{aligned}
$$

Therefore $\hat{G}_{0}(x)$ depends only on the concentration. The series expansion of the generating function can then be expressed using the series expansion of $(1-\alpha x+\beta x)^{1 / 2}=\sum_{n \geq 0} C_{n} x^{n}$ which leads to

$$
\hat{G}_{0}(x)=N^{-1} \hat{P}_{1}(0) x+\frac{1}{2} \sum_{n \geq 2}\left(C_{n+1}-C_{n}\right) x^{n}
$$

from which we can identify the size distribution $\bar{P}_{n}=\frac{1}{2}\left(C_{n+1}-C_{n}\right)$ for $n \geq 2$. Expansion coefficients $C_{n}$ can be written and rearranged as a double sum (see Appendix B), and we obtain the formula given in Eq.(B3)

$$
C_{n}=\frac{(-1)^{1+[n / 2]} \alpha^{n}}{2^{n}(2[(n+1) / 2]-1)} \mathcal{P}_{[n / 2]}^{(-n+1 / 2,-1)}(7 / 9)
$$

where $\mathcal{P}_{[n / 2]}^{(-n+1 / 2,-1)}(z)$ is the Jacobi polynomial which depends on $n$ only and [.] in the integer part. We should distinguish between odd and even $n$ values, and study the asymptotic limit when $n \rightarrow \infty$ (see Appendix B for details). In particular we find that

$$
\begin{aligned}
\bar{P}_{2 m} & =\frac{(-1)^{m} \alpha^{2 m}}{2^{2 m+1}}\left[\frac{\mathcal{P}_{m}^{(-2 m+1 / 2,-1)}(7 / 9)}{2 m-1}-\frac{\alpha \mathcal{P}_{m}^{(-2 m-1 / 2,-1)}(7 / 9)}{4 m+2}\right] \\
& \simeq \frac{A_{0}}{\sqrt{\pi m}}\left(\frac{1-A(c)}{2 m+1}+\frac{2}{4 m^{2}-1}\right) \exp (2 m \ln A(c))
\end{aligned}
$$

where

$$
A(c)=\frac{c(1-c)}{(1+c)(\sqrt{2}-1)^{2}}, A_{0}=\frac{\sqrt{2}-1}{2^{1 / 4}}
$$

and for odd values

$$
\begin{aligned}
\bar{P}_{2 m+1} & =\frac{(-1)^{m} \alpha^{2 m+1}}{2^{2 m+2}(2 m+1)}\left[\frac{\alpha}{2} \mathcal{P}_{m+1}^{(-2 m-3 / 2,-1)}(7 / 9)-\mathcal{P}_{m}^{(-2 m-1 / 2,-1)}(7 / 9)\right] \\
& \simeq \frac{A_{0}}{2 \sqrt{\pi}}\left(\frac{1-A(c)}{\sqrt{m}}+\frac{A(c)}{2 m^{3 / 2}}\right) \frac{\exp [(2 m+1) \ln A(c)]}{2 m+1}
\end{aligned}
$$




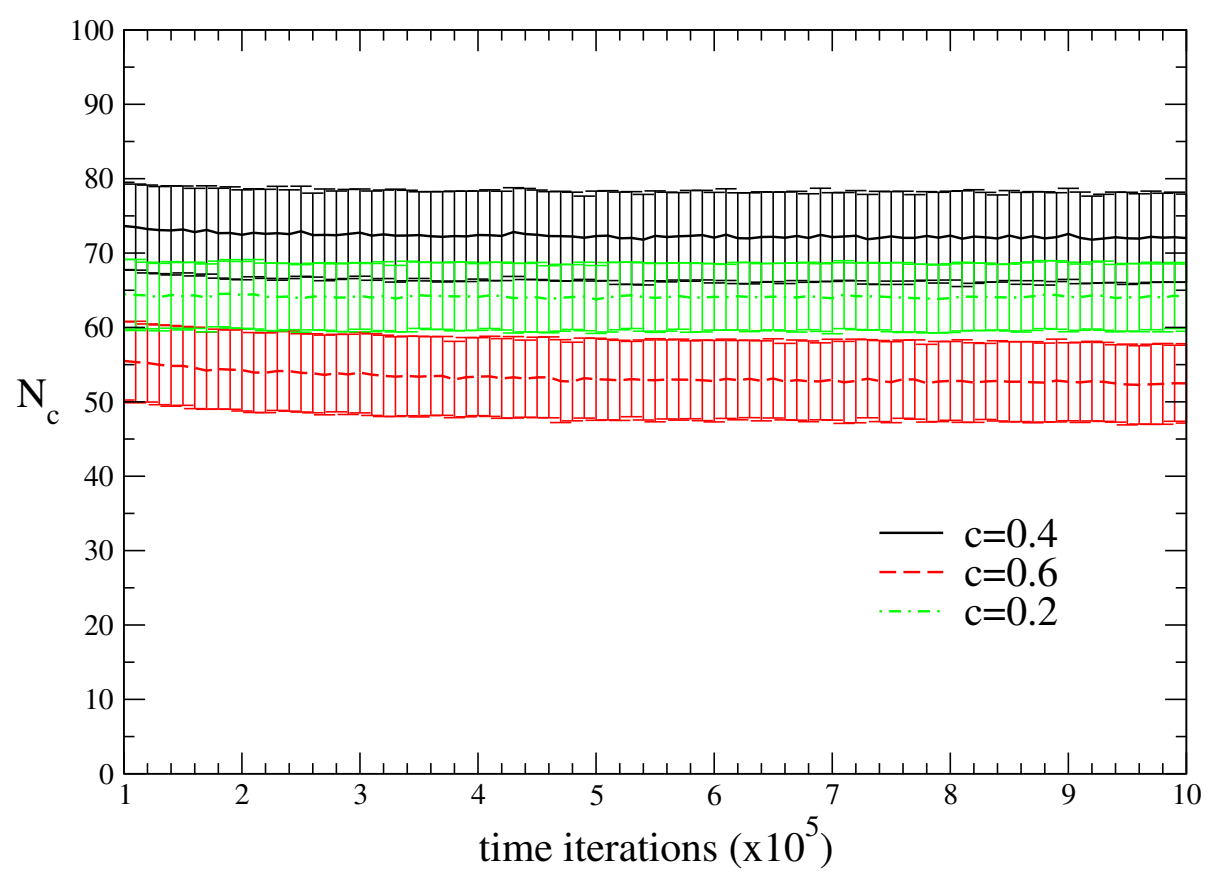

FIG. 2: Average number of clusters with their standard deviation as function of time for $N=500$ and $10^{3}$ initial random configurations.

The quantity $A(c)$ is positive and less than unity. $\bar{P}_{n}$ is exponential with a corrective power law factor $n^{-3 / 2}$, except at the value where $A(c)=1$, which is achieved at the critical point $c^{*}=\sqrt{2}-1$. For this value, $\bar{P}_{n}$ follows a power law with decay exponent $5 / 2$ since the amplitude $1-A(c)$ for the $n^{-3 / 2}$ contribution vanishes, as seen in the previous expressions Eq.(18) and (20). In general, $1-A(c)$ remains a small quantity around $c^{*}$, so that the apparent exponent is close to $5 / 2$. For example $A(0.3) \simeq 0.94$, or $A(0.5) \simeq 0.97$ which is close to unity. This corresponds to $\beta=1$ in the model [11] since $\eta=3 / 2+\beta$ if we identify the two models, and this is represented by an aggregation kernel equal to the size product of its constituents. Also, near $c^{*}, \ln A(c) \simeq-\left(1-c / c^{*}\right)^{2} / \sqrt{2}$, which gives a diverging characteristic cluster size $n_{c}=\sqrt{2}\left(1-c / c^{*}\right)^{-2}$.

Numerically, we simulated the stochastic dynamics using a number of random initial configurations at fixed concentration and system size. A site is chosen randomly with a uniform probability and if it is occupied by a particle, this one is allowed to move either on the left or right by one step with equal probability, provided that the destination site is empty. If the particle moves to the left, it leaves the cluster where it was attached eventually. If the particle moves to the right, it moves with all the cluster it is attached to accordingly. 


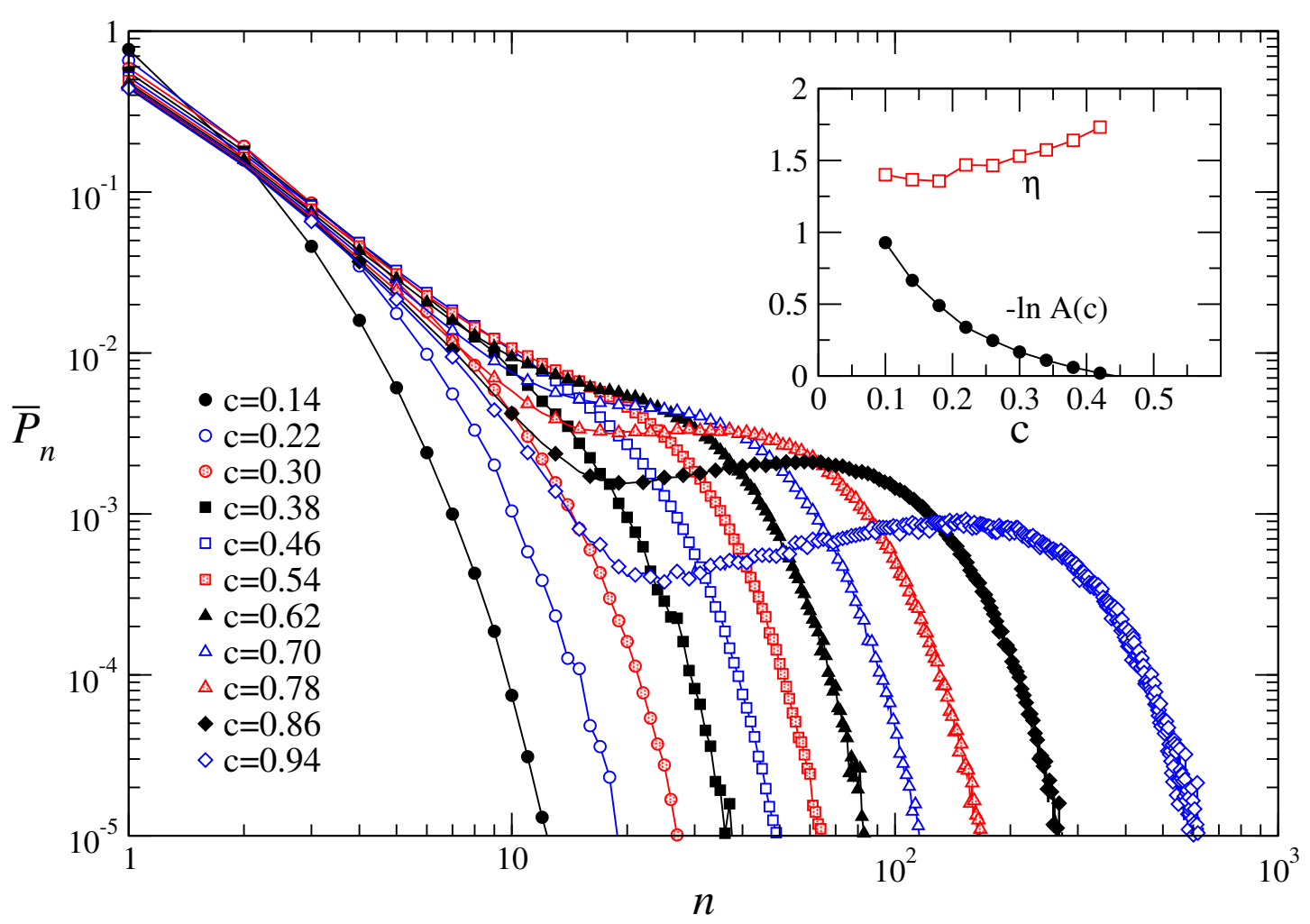

FIG. 3: Numerical evaluation of the cluster size distribution $\bar{P}_{n}$ for $N=2000$ and different concentrations $c .10^{5}$ random initial realisations were generated, and averages were performed after $2 \times 10^{5}$ time steps. In inset, black dot symbols represent coefficient $-\ln A(c)$ of fitted function $\bar{P}_{n} \simeq A(c)^{-n} n^{-\eta}$, and square red symbols are the power law exponent $\eta$.

The process is repeated at each time iteration, and the size distribution is evaluated after the steady state is reached, see Fig. 2, typically after at least $10^{5}$ time steps for the sizes considered in simulations. For different concentration values, the averaged size distribution is displayed in Fig. 3 for $N=2000$. The cutoff at $n \simeq c N$ induces a sharp decrease of the curves at large concentration beyond the power law region for finite $N$. As discussed before, this cutoff is not included in the computation of Eq.(14), since we consider the thermodynamical limit $N \gg 1$. In the inset of the figure we have plotted the parameters for the fitting function $\bar{P}_{n} \simeq A(c)^{-n} n^{-\eta}$ at different concentrations. Using a polynomial fit, $1-A(c)$ appears to vanish for $c \simeq 0.443$, which is close to the theoretical value 0.414 from the approximation method Eq.(14). The exponent $\eta$ is close to $3 / 2$ for most of concentrations around $c^{*}$, which is the exponent extracted from the asymptotic values Eq.(18) and (20). We would normally expect an exponent close to $5 / 2$ in the critical region, due to the smallness 


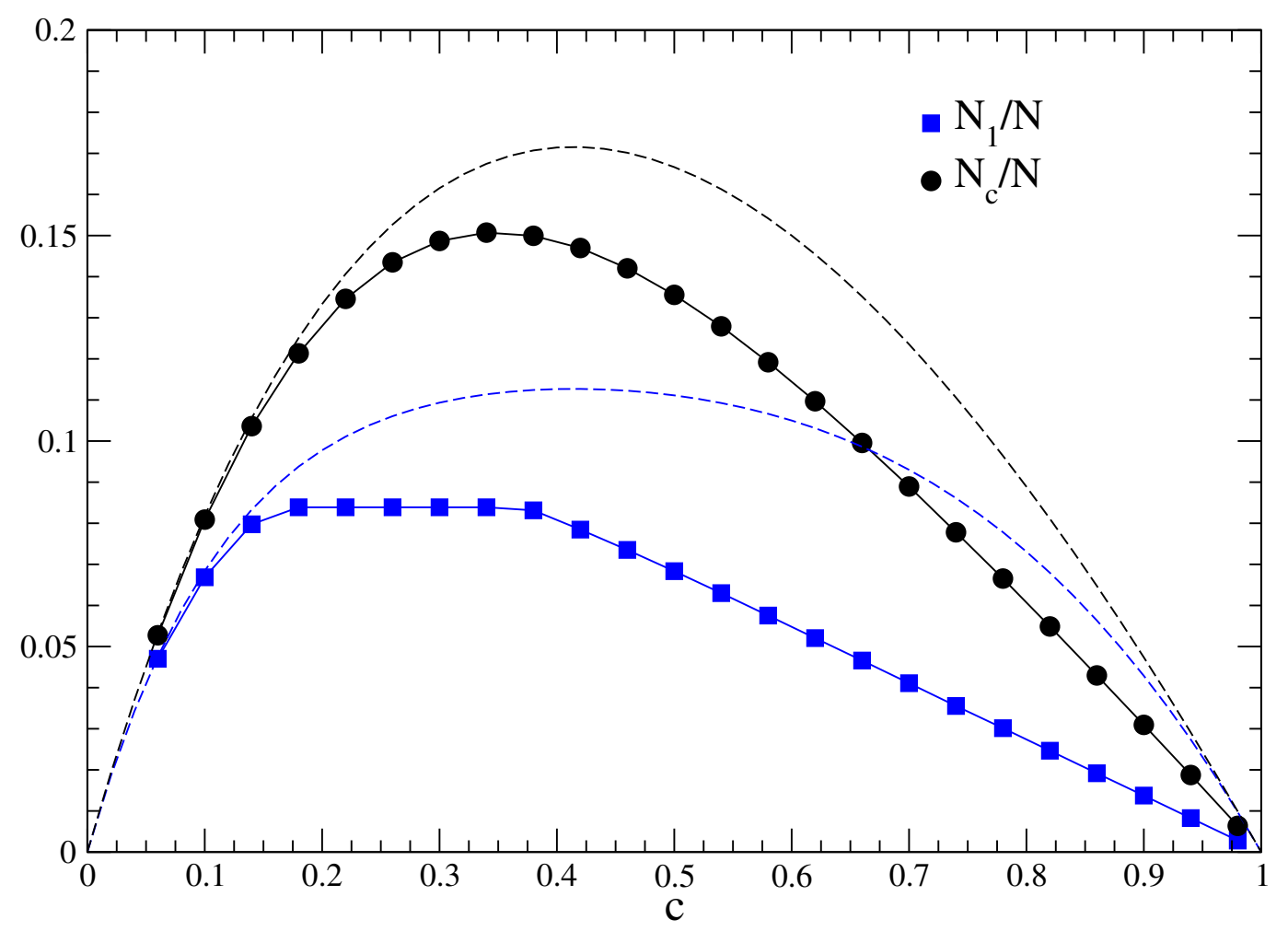

FIG. 4: Numerical average values of one-particle cluster number $N_{1}$ and total cluster number $N_{c}$. $10^{5}$ random initial realisations were generated, and averages were performed after $2 x 10^{5}$ time steps. Dotted lines are the approximated values given by the generating function Eq.(14).

of factor $1-A(c)$. However the mean-field approximation gives a qualitative explanation of the dynamics with the possible numerical evidence of a critical point near $c^{*}$. At higher concentrations $c>c^{*}$, we observe that large clusters tend to aggregate and dominate the dynamics since particles are getting closer to each other, and then merge more easily. Indeed, the finite size effect induces strong contributions to large clustering as $\bar{P}_{n}$ approaches the singular limit $\bar{P}_{n} \simeq \delta_{n, c N}$ when $c \simeq 1$, which is observed in the curves above $c=0.78$ of Fig. 3 by the appearance of a broad peak in the distributions. At low concentrations, the distribution follows mostly an exponential law with a large amplitude $A(c)$ as the clusters tend to diffuse and break into small independent particles. In Fig. 4, we have plotted the average number of clusters $N_{c}$ and the average number of one-particle clusters $N_{1}$ as a function of the concentration. Dotted lines represent the values given by Eq.(14), i.e. $N_{c}=N c(1-c) /(1+c)$ and $N_{1}=\hat{P}_{1}(0)$; see Eq.(15). The agreement is correct for small values of concentrations, and the behavior of $N_{1}$ is modified near $c \simeq 0.4$ above which it 


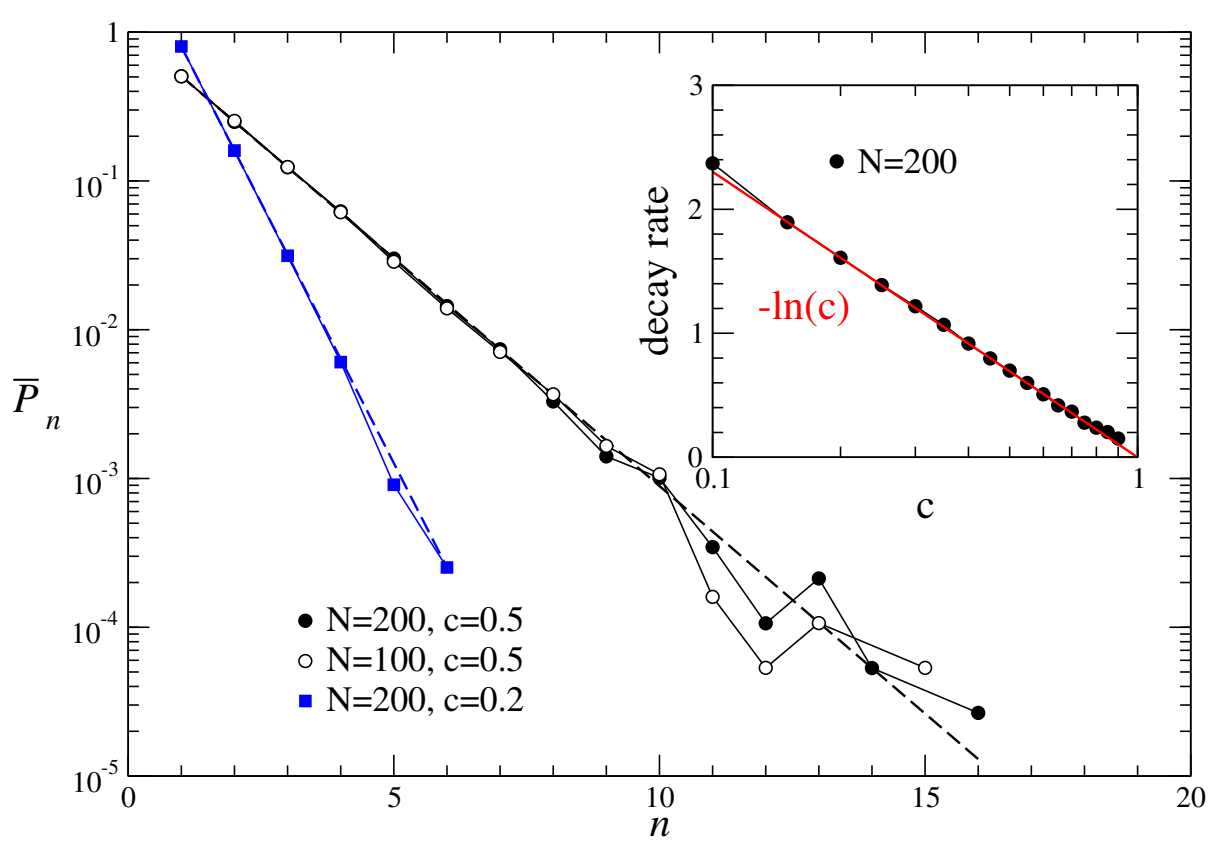

FIG. 5: Averaged size distribution $\bar{P}_{n}$ for a simple particle dynamics and system size $N$ : particles can diffuse to the left or right if the final site is unoccupied. The averaging is evaluated after $10^{6}$ time steps and $10^{3}$ samples. In inset: decay rate as function of the concentration $c$.

seems to follow a linear decreasing law.

For comparison, let us consider the simplest symmetric and local dynamics where particles are allowed to diffuse only on one of the two neighboring sites if these are unoccupied. The averaged size distribution $\bar{P}_{n}$ of the $n$-particle clusters can be evaluated naively as function of the particle concentration $c$. Indeed a cluster of $n$ particles exists with a probability proportional to $(1-c)^{2} c^{n}$, and the averaged distribution follows therefore a simple exponential law $\bar{P}_{n}=(1-c)^{2} c^{n}$ with a decay rate equal to $-\ln c$, as exemplified in Fig. 5 . Moreover the average number of clusters is equal to the semi-circle law $N_{c}=N c(1-c)$. Any cluster can only fragment by releasing one particle at its edges and the distribution is purely exponential with no power law contribution. Therefore the asymmetric and non-local dynamics studied in this paper induces a power law contribution in the distribution. The asymmetry also induces the presence of an internal current to the right direction, with an average particle velocity equal to $v=N^{-1} \sum_{n}(1-n) \bar{P}_{n}=1-N_{c} / N>0$. The velocity is maximum for states with large clusters or at low concentration. Using the approximate value for $N_{c}$, we find that $v=\left(1+c^{2}\right) /(1+c)$. The velocity is minimum at the critical concentration $c^{*}=\sqrt{2}-1$. 


\section{DISCUSSION}

Stochastic dynamics of clusters with a strong asymmetric kinetic rule displays a critical behavior at a finite concentration $c^{*} \simeq 0.414$, where the size-distribution becomes a powerlaw with a decay exponent equal to $5 / 2$. This critical point can be simply viewed as the singular point of the square root term in the generating function Eq.(14) at $x=1$, where the quantity $1-\alpha x+\beta x^{2}$, which is always positive for $0 \leq x<1$, vanishes. It is interesting to compare these results with a model of coagulation with single-particle fragmentation [18] and using the product form for the aggregation kernel, which leads to gelation after a finite time. The mass or size distribution displays, when the gelation time is approached from below, a power law with the same exponent $5 / 2$ at the critical concentration. We can deduce that the present model might belong to a class of models defined by an aggregation kernel proportional to the product of individual masses or sizes. A quasi-gelation state exists if the chain is not periodic and boundary conditions are fixed at both ends, which modifies the stochastic rules at the boundaries. Indeed the current tends to aggregate all particle to the right end of the chain where a large cluster forms since no particle can be transferred to the left hand side of the chain. After a short time, almost all particles belong to the large cluster, with few small clusters surviving in the steady state. This may be in analogy with percolation problems in higher dimensions where small clusters persist after the spanning cluster is formed.

Corrections to the theory of the present model would incorporate finite size cutoff of the cluster sizes at $n=c N$ in Eq.(14), which would explain the broad contribution of large clusters at higher concentrations in Fig. 3. Also, we need to take into account the momenta contributions $k \neq 0$ in Eq.(12) in order to give more accurate results, for example by considering fluctuations around the uniform solution: $\hat{G}(x, k)=N \delta_{k, 0} \hat{G}_{0}(x)+\delta G(x, k)$ with $\delta G(x, 0)=0$. This latter substitution would however not modify the average value of the cluster number as non-linear effects should be important. We should also ask whether the critical point is robust when we modify the fragmentation rule. For example Eq.(8) can be generalized to any fragmentation distribution, for example we could impose than more that one particle can break-off from the cluster from the left. In this context, there should be a class of fragmentation rules that allows the existence of the critical point.

This work was supported by Brain Pool Program through the National Research Foun- 
dation of Korea (Grant No. NRF-2018H1D3A2065321.

\section{APPENDIX A: BALANCE EQUATIONS FOR THE DIFFERENT PROCESSES}

In this section, we derive the explicit expressions for the different processes that contribute to the master equation Eq.(8). For the process $(a)$, one has simply

$$
\operatorname{Prob}\left(\stackrel{\curvearrowleft}{\circ} \cdots^{r+n-1} \bullet\right)=\operatorname{Prob}\left(\circ \bullet^{r} \ldots{ }^{\stackrel{r+n-1}{\bullet}} \circ\right)=\frac{1}{\tau} P_{n}(r)
$$

For the process $(b)$ one has instead

$$
\begin{aligned}
\operatorname{Prob}\left(\times \bullet \circ \bullet^{r} \ldots{ }^{r+n-1}\right. & \bullet \\
& =\frac{1}{\tau} P_{n}(r) \times\left[P_{1}(r-2)+P_{2}(r-3)+\cdots+P_{N_{p}-n}\left(r-N_{p}+n-1\right)\right](\mathrm{A} 2)
\end{aligned}
$$

Process $(c)$ is given by

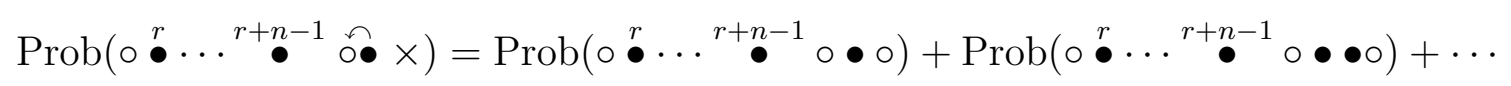

$$
\begin{aligned}
& =\frac{1}{\tau} P_{n}(r) \times\left[P_{1}(r+n+1)+P_{2}(r+n+1)+\cdots+P_{N_{p}-n}(r+n+1)\right](A 3
\end{aligned}
$$

Process $(d)$ is given by

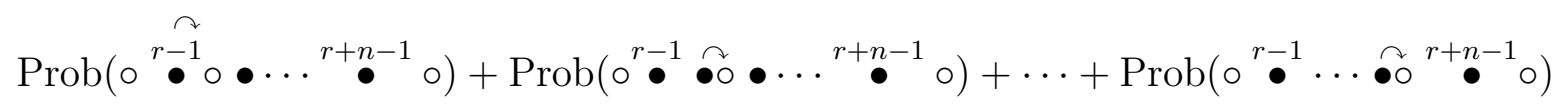

$$
\begin{aligned}
& =\frac{1}{\tau}\left[P_{1}(r-1) P_{n-1}(r+1)+P_{2}(r-1) P_{n-2}(r+2)+\cdots+P_{n-1}(r-1) P_{1}(r+n-1)\right]
\end{aligned}
$$

Process $(e)$ is given by

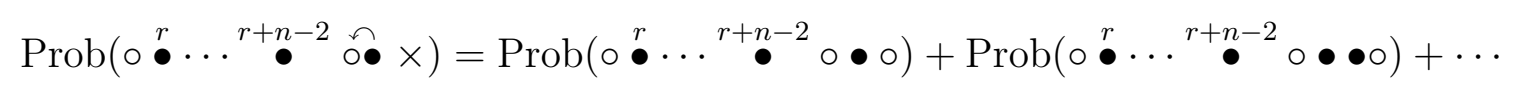

$$
\begin{aligned}
& =\frac{1}{\tau} P_{n-1}(r) \times\left[P_{1}(r+n)+P_{2}(r+n)+\cdots+P_{N_{p}-n}(r+n)\right](
\end{aligned}
$$

Process $(f)$ is given by

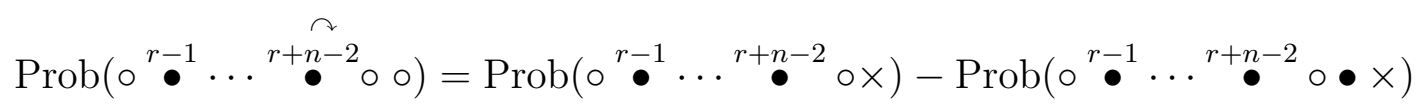

$$
\begin{aligned}
& =\frac{1}{\tau} P_{n}(r-1) \times\left[1-P_{1}(r+n)-P_{2}(r+n)-\cdots-P_{N_{p}-n}(r+n)\right]
\end{aligned}
$$

And the last process $(g)$ is given by

$$
\operatorname{Prob}\left(\circ \stackrel{\curvearrowleft}{r-1} \bullet \cdots{ }^{r+n-1} \bullet\right)=\frac{1}{\tau} P_{n+1}(r-1)
$$




\section{APPENDIX B: EXPANSION COEFFICIENTS $C_{n}$}

We consider in this appendix the expression of coefficients $C_{n}$ in Eq.(16), and their behavior when $n$ is asymptotically large. From Eq.(14), we first have to expand the quantity

$$
\begin{aligned}
\sqrt{1-\alpha x+\beta x^{2}} & =\sum_{n \geq 0} a_{n}\left(-\alpha x+\beta x^{2}\right)^{n}=\sum_{n \geq 0} a_{n} x^{n} \sum_{k=0}^{n}\left(\begin{array}{l}
n \\
k
\end{array}\right)(-\alpha)^{n-k} \beta^{k} x^{k} \\
a_{n} & =\frac{(-1)^{n+1}(2 n) !}{2^{2 n} n !^{2}(2 n-1)}
\end{aligned}
$$

We can rearrange the terms in the double sum so that

$$
\begin{aligned}
\sum_{n \geq 0} a_{n} x^{n} \sum_{k=0}^{n}\left(\begin{array}{l}
n \\
k
\end{array}\right)(-\alpha)^{n-k} \beta^{k} x^{k} & =\sum_{n \geq 0} x^{n} \sum_{k=0}^{[n / 2]} a_{n-k}\left(\begin{array}{c}
n-k \\
k
\end{array}\right)(-\alpha)^{n-2 k} \beta^{k} \\
& =\sum_{n \geq 0} x^{n}(-\alpha)^{n} \sum_{k=0}^{[n / 2]} a_{n-k}\left(\begin{array}{c}
n-k \\
k
\end{array}\right) 36^{-k}=\sum_{n \geq 0} C_{n} x^{n}
\end{aligned}
$$

For $n$ even or odd respectively, the sums over $k$ have a representation in term of Jacobi polynomials

$$
\begin{aligned}
C_{2 m} & =\frac{(-1)^{m+1} \alpha^{2 m}}{4^{m}(2 m-1)} \mathcal{P}_{m}^{(-2 m+1 / 2,-1)}\left(z_{0}\right), \\
C_{2 m+1} & =\frac{(-1)^{m+1} \alpha^{2 m+1}}{4^{m}(4 m+2)} \mathcal{P}_{m}^{(-2 m-1 / 2,-1)}\left(z_{0}\right)
\end{aligned}
$$

with $z_{0}=7 / 9$. For $m$ large, we would like to obtain an expansion of the polynomials $\mathcal{P}_{m}^{(-2 m \pm 1 / 2,-1)}\left(z_{0}\right)$. Although there are asymptotic formulas in the literature for a large parameter $m \gg 1[25,26]$, there is no result for the value $z_{0}=7 / 9$ which is outside the domain of convergence of the asymptotic series. We will use instead an integral representation of the polynomials in the complex plane and perform a simple saddle point analysis. If $w(z)=(1-z)^{-2 m \pm 1 / 2}(1+z)^{-1}$ is the weight of the Jacobi polynomials in Eq.(B3), we have

$$
\mathcal{P}_{m}^{(-2 m \pm 1 / 2,-1)}\left(z_{0}\right)=\frac{(-1)^{m}}{2^{m} w\left(z_{0}\right)} \oint \frac{d z}{2 i \pi} \frac{w(z)\left(1-z^{2}\right)^{m}}{\left(z-z_{0}\right)^{m+1}}
$$

where the contour is a small circle around $z_{0}$. A change of variable $z=z_{0}-\left(1-z_{0}\right) s$ leads to

$$
\mathcal{P}_{m}^{(-2 m \pm 1 / 2,-1)}\left(z_{0}\right)=\frac{\left(1+z_{0}\right)^{m}}{2^{m}} \oint \frac{d s}{2 i \pi s^{m+1}} \frac{(1+s)^{ \pm 1 / 2}}{1-r s}\left(\frac{1-r s}{1+s}\right)^{m}
$$

where $r=\left(1-z_{0}\right) /\left(1+z_{0}\right)$ and the contour is a small circle around the origin. For $m$ large, we apply the saddle point method by extremizing the function $\phi(s)=\ln [(1-r s) / s(1+s)]$. 
We obtain the relevant solution $s^{*}=(1-\sqrt{1+r}) / r$ and expand $\phi(s)$ around $s^{*}$ up to the second order, then compute the resulting Gaussian integral. We obtain

$$
\mathcal{P}_{m}^{(-2 m \pm 1 / 2,-1)}\left(z_{0}\right) \simeq-\frac{\left(1+s^{*}\right)^{ \pm 1 / 2}}{s^{*}\left(1-r s^{*}\right)} \frac{\exp \left(m \phi\left(s^{*}\right)+m \ln \left(1+z_{0}\right)-m \ln 2\right)}{\sqrt{2 \pi m \phi^{\prime \prime}\left(s^{*}\right)}}
$$

with the values

$$
\begin{aligned}
\phi\left(s^{*}\right) & =i \pi-\ln \left(1+z_{0}\right)+2 \ln \left(\sqrt{2}+\sqrt{1+z_{0}}\right), \\
\phi^{\prime \prime}\left(s^{*}\right) & =\frac{2^{1 / 2}\left(1-z_{0}\right)^{2}}{\left(1+z_{0}\right)^{1 / 2}} \frac{\left(3+z_{0}-2^{3 / 2} \sqrt{1+z_{0}}\right)}{\left(\sqrt{2}-\sqrt{1+z_{0}}\right)^{4}}
\end{aligned}
$$

Therefore, the asymptotic behavior of $\mathcal{P}_{m}^{(-2 m+\epsilon / 2,-1)}\left(z_{0}\right)$, with $\epsilon= \pm 1$, and which is valid at least in the interval $0<z_{0}<1$, is equal to

$$
\mathcal{P}_{m}^{(-2 m+\epsilon / 2,-1)}\left(z_{0}\right) \simeq \frac{(-1)^{m}\left(\sqrt{2}-\sqrt{1+z_{0}}\right)^{1+\epsilon / 2}\left(1+z_{0}\right)^{1 / 4} e^{m\left(2 \ln \left(\sqrt{2}+\sqrt{1+z_{0}}\right)-\ln 2\right)}}{2^{(5-\epsilon) / 4}\left(1-z_{0}\right)^{\epsilon / 2} \sqrt{3+z_{0}-2^{3 / 2} \sqrt{1+z_{0}}} \sqrt{\pi m}}
$$

where we have, in this limit

$$
\begin{aligned}
\mathcal{P}_{m}^{(-2 m+1 / 2,-1)}\left(z_{0}\right) & \simeq\left(1+s^{*}\right) \mathcal{P}_{m}^{(-2 m-1 / 2,-1)}\left(z_{0}\right) \\
& \simeq \frac{\sqrt{2}\left(\sqrt{2}-\sqrt{1+z_{0}}\right)}{1-z_{0}} \mathcal{P}_{m}^{(-2 m-1 / 2,-1)}\left(z_{0}\right)
\end{aligned}
$$

The asymptotic behavior Eq.(B8) has been checked with (C)Maple2018 for $z_{0}=7 / 9$ with a relative error of about $0.4 \%$ up to $m=80$.

\section{REFERENCES}

[1] Tamás Vicsek and Fereydoon Family. Dynamic scaling for aggregation of clusters. Phys. Rev. Lett., 52(19):1669-1672, 1984.

[2] C. M. Sorensen, H. X. Zhang, and T. W. Taylor. Cluster-size evolution in a coagulationfragmentation system. Phys. Rev. Lett., 59(3):363-366, 1987.

[3] Paul Meakin and Matthieu H. Ernst. Scaling in aggregation with breakup simulations and mean-field theory. Phys. Rev. Lett., 60(24):2503-2506, 1988.

[4] Z Cheng and S Redner. Kinetics of fragmentation. J. Phys. A Math. Gen, 23:1233-1258, 1990. 
[5] Jonathan A.D. Wattis. An introduction to mathematical models of coagulation-fragmentation processes: A discrete deterministic mean-field approach. Physica D, 222:1-20, 2006.

[6] A. E. Kyprianou, S. W. Pagett, and T. Rogers. Universality in a class of fragmentationcoalescence processes. Ann. Inst. Henri Poincaré, B, 54(2):1134-1151, 2018.

[7] Anna S. Bodrova, Vladimir Stadnichuk, P. L. Krapivsky, Jürgen Schmidt, and Nikolai V. Brilliantov. Kinetic regimes in aggregating systems with spontaneous and collisional fragmentation. J. Phys. A Math. Theor., 52(20):205001, 2019.

[8] R. Dennis Vigil, Isaac Vermeersch, and Rodney O. Fox. Destructive aggregation: Aggregation with collision-induced breakage. J. Colloid Interface Sci., 302(1):149-158, 2006.

[9] Jianhong Ke, Zhenquan Lin, and Xiaoshuang Chen. An analytical solution of coagulation processes with collision-induced fragmentation. J. Phys. A Math. Theor., 41(28):285005, 2008.

[10] M. Smoluchowski. Drei vorträge über diffusion, brownsche bewegung und koagulation von kolloidteilchen. Physik. Zeit., 17:557-585, 1916.

[11] H. White Warren. On the Form of Steady-State Solutions to the Coagulation Equations. J. Colloid Interface Sci., 87(1):204-208, 1981.

[12] Stephan M Dammer. Stochastic many-particle systems with irreversible dynamics. PhD thesis, Universität Duisburg-Essen, 2004.

[13] Hisao Hayakawa and Satio Hayakawa. Power-Law Size Distribution of Dust Grains. Publ. Astron. Soc. Japan, 40:341-345, 1988.

[14] Nikolai Brilliantov, P. L. Krapivsky, Anna Bodrova, Frank Spahn, Hisao Hayakawa, Vladimir Stadnichuk, and Jürgen Schmidt. Size distribution of particles in Saturn's rings from aggregation and fragmentation. Proceedings of the National Academy of Sciences, 112(31):9536-9541, 2015.

[15] R. Botet and R. Jullien. Size distribution of clusters in irreversible kinetic aggregation. J. Phys. A, 17(12):2517-2530, 1984.

[16] E. Ben-Naim and P. L. Krapivsky. Condensates in driven aggregation processes. Phys. Rev. E, 75:011103, 2007.

[17] R Dennis Vigil. On equilibrium solutions of aggregation-fragmentation problems. J. Colloid Interface Sci., 336(2):642-647, 2009.

[18] R Dennis Vigil, Robert M Ziff, and Binglin Lu. New universality class for gelation in a system with particle breakup. Phys. Rev. B, 38(1):942, 1988. 
[19] Hans Babovsky. Gelation of stochastic diffusion-coagulation systems. Physica D: Nonlinear Phenomena, 222:54-62, 2006.

[20] A. A. Lushnikov. Gelation in coagulating systems. Physica D: Nonlinear Phenomena, 222:37$-53,2006$.

[21] Takahiro Ezaki and Katsuhiro Nishinari. Cluster size distribution in 1D-CA traffic models. $J$. Phys. A: Math. Theor., 45(4):045101, 2012.

[22] O. Pulkkinen and J. Merikoski. Cluster size distributions in particle systems with asymmetric dynamics. Phys. Rev. E, 64(5):056114, 2001.

[23] H. Hayakawa. Irreversible kinetic coagulations in the presence of a source. J. Phys. A, 20:L801-L805, 1987.

[24] Colm Connaughton, R Rajesh, and Oleg Zaboronski. Kinetics of Cluster-Cluster Aggregation, chapter 16, pages 1-17. CRC, Boca Raton, 1999.

[25] Z. Song and R. Wong. Asymptotics of Pseudo-Jacobi Polynomials with Varying Parameters. Stud. Appl. Math., 139(1):179-217, 2017.

[26] Roderick Wong. Asymptotics of orthogonal polynomials. Int. J. Numer. Anal. Model., 15(1):193-212, 2018. 